\title{
Traditional Indian Medicine (TIM) based Humor Identification - A Recurrence based Approach
}

\author{
Prasad Joshi \\ Research Scholar \\ Veermata Jijabai Technological \\ Inst., Mumbai, India
}

\author{
Kiran Ambekar \\ Practicing Physician \\ Mumbai, India
}

\author{
Rohin Daruwala \\ Professor, Electrical Eng. Dept., \\ Veermata Jijabai Technological \\ Inst., Mumbai, India
}

\begin{abstract}
Objective analysis and interpretation of radial pulse signal has been attempted. Capacitance variation based sensing and instrumentation mechanism has been developed for pulse sensing and logging. Traditional Indian Medicine (TIM) perspective for humor identification has been attempted. Pulse signal of healthy individual analysed in dominant humor interval, display prominent changes in recurrence quantification measures for pulse amplitude variations. These marker results are observed to correlate strongly with those obtained for a group of healthy individuals in a dominant humor interval period. Recurrence quantification analysis can possibly help in arriving at inferences apparent to sense perception for a TIM physician.
\end{abstract}

\section{General Terms}

Biomedical Signal Processing, Traditional Indian Medicine(TIM) - Ayurveda.

\section{Keywords}

Radial Pulse Analysis, Recurrence Quantification Analysis and Humor Identification.

\section{INTRODUCTION}

An important tool for patient diagnosis in the system of Traditional Indian Medicine (TIM) is examining the radial pulse $[1,2]$. The process involves the physician feeling the patient's radial pulse using his three fingers viz. the index, middle and the ring, to evaluate the extent of $\operatorname{Vata}(V t)$, $\operatorname{Pitta}(P t)$ and Kapha(Kp) dosha(energy/humor) in the patient. Although the procedure is subjective and highly skill based, quite a few practicing physicians make a reasonably accurate diagnosis, verified and corroborated by pathological tests [1, 2]. The present study, similar to $[3,4,5,6,7]$ attempts to objectively explore and analyze the radial pulse signal obtained from along the radial artery, for exploring the temporal and spatial domain dynamics.

As reported in 'Annexure IX' and 'Summary of Infrastructure Facilities' appearing in [8], there are 4,78,750 registered Ayurveda (TIM) practitioners in India across all the states. A total of $8,31,15,139+6,55,390$ patients, in OPD \& IPD, visited over the country, across various hospitals for Ayurvedic consultations in the year 2010-11[8]. A TIM physician relies on pulse examination, apart from the conventional patient examination methodologies, for ailment diagnosis[9]. Pulse examination involves, identification and quantification of Vata $(\mathrm{Vt})$, Pitta $(\mathrm{Pt})$ and Kapha $(\mathrm{Kp})$, energies/humors, as the principle deciding factors for patient diagnosis[chapter 01 in 1, chapter 05 in 2]. Conventionally TIM physicians solely rely on sense perception for $\mathrm{Va}, \mathrm{Pt}$ and $\mathrm{Kp}$ identification and quantification. The present study, similar to earlier $[10,11,12]$, attempts to identify signal processing based measures/ parameters for identifying Vt, $\mathrm{Pt}$ and Kp humors for achieving an objective outlook. This can help in the realisation of a low cost, non-invasive diagnostic tool as a value added aid for physicians.

The present paper is divided in the following parts $\S(2)$ About TIM. §(3)Pulse sensing mechanism. §(4)Pulse analysis: Tools $\&$ Premise. §(5) Results and discussions. §(6) Conclusions.

\section{ABOUT TRADITIONAL INDIAN MEDICINE (TIM)}

As detailed extensively in the seminal works [1, 2, 13], Traditional Indian Medicine (TIM) - Ayurveda, essentially considers the body, mind and soul as a single entity or the living being. Air, Water, Earth, Fire and Space or Ether are considered as the constituent building blocks in the composition of all living matter and express themselves in the form of combination identifiers qualified as 'doshas' (energy/humor). These humor identifiers, or the primary controlling forces are composed of - Vata (Space and Air), Pitta (Fire and Water) and Kapha (Earth and Water). In the state of balance and well-being the three humors are maintained in normalized proportion, relative to the specific individual. The above humors can also be considered as energies, each being the driving force responsible for some or the other metabolic process. Extensive details of the nature and characteristic of the three type of humor are reported in [chapter $01 \& 03$ in 1, chapter 08 in 2, chapter 03 in 13]. Vata (Vt) is the energy of movement or activity including the extensive communication network of the body. Pitta $(\mathrm{Pt})$ is the energy of metabolism or transformation involved in the bodily process of assimilation - whether it is the food ingested or comprehension of other sense perception. Kapha (Kp) is the cohesive energy of the body enjoining and lubricating the tissues and organs. Although no physiological or pathophysiological, empirical evidence exists for practically identifying and quantifying the three humors, their existence in the form of bodily functions can be experienced.

TIM considers every individual to be born with unique composition and combination of the three humors. The nature of one's constitution is decided at birth, however it goes through cyclic changes during the progression of the day, changes in seasons as well as over the period of one's life span. Thus considering a 12-hr periodic cycle, consisting of six intervals of 2-hrs, TIM accounts the first two intervals (4hrs.) in the morning, $6 \mathrm{am}-10 \mathrm{am}$, as the period of Kp. Following this period, the next three intervals (6-hrs.), $10 \mathrm{am}$ $-4 \mathrm{pm}$, are those of $\mathrm{Pt}$, which is followed by a single interval of $\mathrm{Vt}, 4 \mathrm{pm}-6 \mathrm{pm}$. 


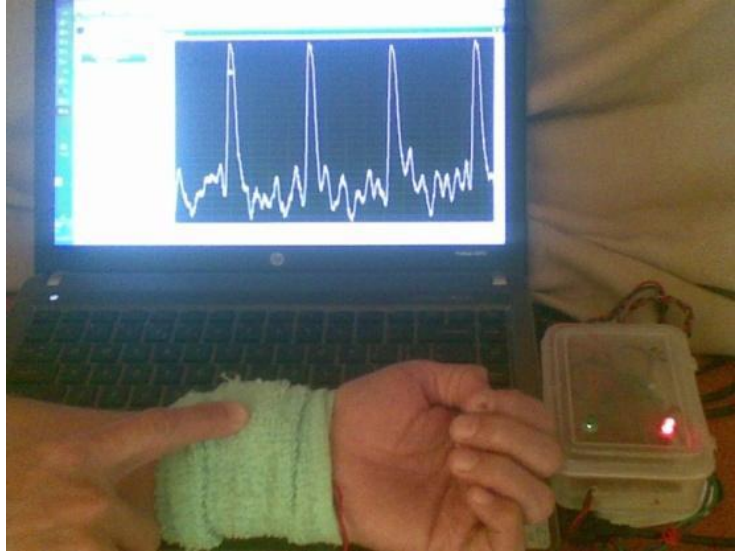

Figure 1: Pulse sensing mechanism.

The 12-hrs. cycle now continues from $6.00 \mathrm{pm}$ in the evening to 6.00 am the next day. This biological cycle can be considered as the circadian cycle [chapter 03 in 1].

TIM literature[1,2,13], identifies and distinguishes radial pulse not only on the basis of rate and amplitude, but essentially on the basis of contour variations, as felt to sense perception and described in terms of gait/movements of various animals. Characterising features for the three humor, as reported in literature[ chapter $01 \& 03$ in 1 , chapter 08 in 2], which assist a TIM physician in identifying them are: Kp humor pulse - associated with the movement of swan, slow in rate and reported to be 'deep', 'cool', and with 'moderate tension'; Pt humor pulse - jerky like frog movement with moderate rate, 'strong', hot' \& 'prominent' and 'high in tension'; Vt humor pulse: is likened to movement of snake, 'fast', 'cold', and 'low in tension' but 'irregular' in rate. Thus objectively it can be inferred that radial pulse contains information in tempro-spatial domain, identified by patterns as perceived to the sense perception.

\section{PULSE SENSING MECHANISM}

The radial pulse signal is a pressure pulse signal as measured at the root of the aorta, characterized by a rising pressure wave having a dome like top terminated by a notch called as the incisura, and followed by a decreasing pressure waveformtrough, corresponding to the systole [14]. Contour of the pressure pulse waveform as recorded at the base of aorta changes significantly with that of the pressure pulse waveform as measured at the radial pulse position. Changes in contour shape as well as intensity of the pressure pulse waveform are caused due to decreasing arterial/capillary diameters, decreasing pressure and are also contributed by the total distensibility of the arterial tree [14]. On lines similar to authors previous works $[10,11,12]$, a capacitance based pulse sensing mechanism has been used for signal sensing (Figure 1). An electret capacitive pick-up (typical CMA-4544PF-W available from DigiKey part no 102-1721-ND), and having a near flat frequency response up-to $20 \mathrm{kHz}$ is used for sensing the radial pulse perturbations. Sensed signal is, sampled at $500 \mathrm{~Hz}$. and digitised using a 14-bit DAQ, USB-6009 from National Instruments. The above sensing mechanism and instrumentation has helped us validate results of heart rate variability (HRV) using pulse rate variability (PRV) techniques[10, 12]. Logged pulse signals are hardthresholded to detect signal peaks. After eliminating outliers due to false peak detection and motion artifacts, pulse peak-topeak amplitude series - PPa, is obtained.
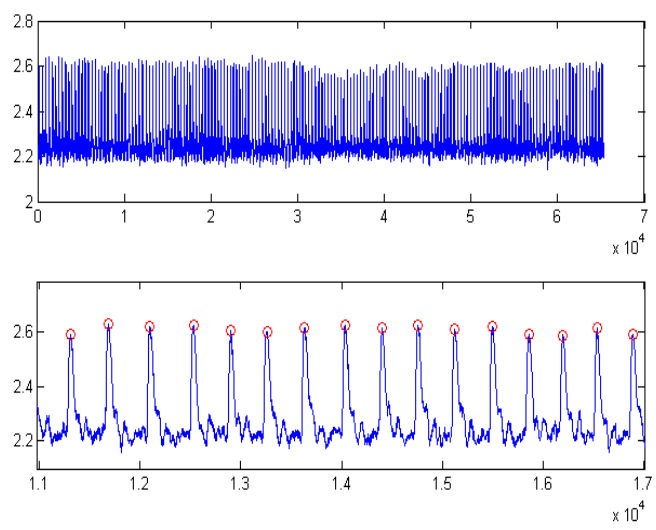

Figure 2: Sample sensed signal, along-with peaks identified \& highlighted, (amplitude(V) versus sample index (n).

\section{PULSE ANALYSIS: TOOLS AND PREMISE}

As detailed in [15], time series generated from biological processes contain deterministic as well as stochastic components, thus exploring both the approaches provides complementary information in signal analysis. Deterministic approach of analysis involves the use of Taken's theorem for resolving the two dimensional time series in n-dimensions to observe system dynamics and arrive at measures for quantifying the n-domain phase space plot. Stochastic approach observes the series in its native/expressed timeseries form and arrives at entropy measures for quantifying the dynamics in terms of rate of information generation. In the present work the radial pulse, peak-peak amplitude series $\mathrm{PPa}$, has been analysed for exploring amplitude variation using recurrence quantification analysis.

\subsection{Recurrence Quantification Analysis}

Recurrence plot (RP) is a graphical tool to visualise the state of a dynamic system and is especially used to observe and evaluate the non-stationarity in the analysed signal $[16,17]$. As defined and described in [16], RP essentially renders a multidimensional phase-space trajectory representation of a system, with embedded dimension $\mathrm{m}$, into a two dimensional distance matrix consisting of 1's and 0's, obtained on the basis of presence or absence of nearest neighbors lying within the threshold distance $(\epsilon)$, which can be expressed as

$$
R_{i, j}(\epsilon)=\Theta\left(\epsilon-\left\|x_{i}-x_{j}\right\|\right) \quad i, j=1 \ldots N
$$

where $\mathrm{N}$ is the number of measured points $x_{i}, \boldsymbol{\epsilon}$ is the threshold distance, $\Theta(\cdot)$ the Heaviside function such that $\boldsymbol{\Theta}(\boldsymbol{x})=\mathbf{0}$ if $\boldsymbol{x}<0$ and $\boldsymbol{\Theta}(\boldsymbol{x})=\mathbf{1}$ otherwise, and $\|\cdot\|$ is a norm. The m-dimensional phase space rendering of a single dimensional time series $x_{i}$ is obtained using the time delay method of Taken's theorem [16]. Thus using RP, the N-point discrete time sequence $x_{i}$ is expressed as a, N-(m-1) X N-(m1) distance matrix, rendered binary, by the threshold distance $\boldsymbol{\epsilon}$.

Since recurrence is a dominant characteristic of many dynamically changing systems, it can be used in the form of plot as well as quantifiable parameters, for obtaining an insight into the systems dynamics. Recurrence plot based measures are relevantly suited for short and non-stationary data patterns [16]. In light of the characteristic features describing the three humors of $\mathrm{Vt}, \mathrm{Pt}$ and $\mathrm{Kp}$, and the cyclic changes displaying the recurrence behavior of these humors, 
recurrence quantification analysis (RQA) is used for observing the recurrence feature in radial pulse amplitude signal and correlate them with those expected by TIM literature.

\subsection{Analysis Methodology}

Considering the individual-centric approach - 'prakruti' stressed by TIM and as elucidated in [chapter 02 in 1 , chapter 07 in 13] and to address benchmarking and validations of results, pulse signals of single individual, viz. author $1-42$ years, male, no prior ailment history - were considered. Pulse signal from point position proximal to the thumb, and along the radial artery, of the right/left hand were identified for single-channel pulse sensing.

The signal was logged for at-least 5-minute duration, from the indicated point position, in the three humor interval periods at $7 \mathrm{am}, 1 \mathrm{pm}$ and $5 \mathrm{pm}$. The humor interval specific radial pulse signals are identified as, K_pulse, P_pulse and V_pulse respectively. Pulse logging activity for the healthy individual in three interval period was carried over an eight-day period as the subject went about the day to day activities. Measures computed for these are now considered as markers or reference. These measures are correlated with results obtained from a group of healthy individuals for examining correlation-ship if any with one of the dominant humors.

\subsection{Phase-Space Analysis of PPa}

Phase space trajectory reconstruction for obtaining recurrence plot involves primarily time-delay based reconstruction with specified embedding dimension - $\mathrm{m}$. With pulse peak-to-peak amplitude series - PPa being considered, amplitude trajectory with m-dimensions can hence be evaluated. It gives a deterministic equivalent for contour dependent conditions as described for various humors in TIM.

In the present study RP based quantification measures applied to the K_pulse, P_pulse, and V_pulse, have been computed, with at-least 250 pulse peaks processed, using Matlab ${ }^{\circledR}$ based tools available in [18]. RQA parameter values decided using [16][17][19] are - embedding dimension(m) - 10; delay - 1 (for discrete time systems); maximum neighborhood;

threshold value $(\epsilon)-0.9$, which is $15 \%$ of maximal phase space diameter as recommended by [20]; line length - 2 for diagonal and vertical line.

\subsubsection{Recurrence Quantification Analysis (RQA) \\ Measures}

Diagonal and vertical line structures form the basis for quantifying recurrence plot and are called as recurrence quantification analysis(RQA) measures. RQA measures, as defined in [16] and computed for the PPa series, using [18] are:

Recurrence Rate (RR) - ratio of recurrent points to the total number of points - identifies the plot density, and is defined as:

$$
R R(\varepsilon)=\frac{1}{N^{2}} \sum_{i, j=1}^{N} R_{i, j}(\varepsilon)
$$

where $\mathrm{N}$ is the total number of sample points under consideration and $\varepsilon$ is the threshold distance.
Determinism (DET) - ratio of recurrent points contributing to diagonal line structures to the total number of recurrent points defined as:

$$
D E T=\frac{\sum_{l=l_{\min }}^{N} l P(l)}{\sum_{l=1}^{N} l P(l)}
$$

where $\boldsymbol{P}(\boldsymbol{l})$ is the frequency of occurance of diagonal line of length $\boldsymbol{l}$, formed by recurrent points at threshold distance $\boldsymbol{\epsilon}$.

Lmean, Lmax - mean \& maximum diagonal line length of the plot, with the average line length defined as:

$$
L=\frac{\sum_{l=l_{\min }}^{N} l P(l)}{\sum_{l=l_{\min }}^{N} P(l)}
$$

Entropy (ENTR), is measured in terms of probability, $p(l)=P(l) / N_{l}$, of finding diagonal line of specified length $l$ in the plot i.e. plot complexity in terms of diagonal line structures and is defined as:

$$
E N T R=-\sum_{l=l_{\min }}^{N} p(l) \ln (p(l))
$$

Laminarity(LAM) - On lines similar to DET, laminarity is defined as the ratio of recurrent points contributing to vertical lines to the total number of recurrent points, defined as:

$$
L A M=\frac{\sum_{v=v_{\min }}^{N} v P(v)}{\sum_{v=1}^{N} v P(v)}
$$

where $P(v)$ is the frequency of occurance of vertical line of length $v$.

Trapping Time(TT) - is the average vertical line length and is defined as:

$$
T T=\frac{\sum_{v=v_{\min }}^{N} v P(v)}{\sum_{v=v_{\min }}^{N} P(v)}
$$

with the maximum length of vertical line identified as $V \max$.

\section{RESULTS AND DISCUSSIONS}

It can be inferred form, Table 1 that, recurrence rate (RR\%) for the amplitude variations of the pulse signal in the three intervals is reasonably low, indicating presence of large white areas which may convey non-stationarity or occurrence of rare states far from normal [16]. However, given the fact that pulse signal in healthy state are being examined, it is required to inspect the measures in Table 1 on the basis of values of a given humor and its identifying features as well as relative variation between the values of three humors. Nevertheless high level of determinism indicates the fact that maximum recurrent points contribute to diagonal or vertical line structures. Whereas diagonal line structures in RP result due to recurrence of segment structures in the phase-space trajectory over a period of time, the length of the diagonal conveys the time over which the trajectory path evolved/occurred recurrently [16]. Kp humor has highest mean and maximum diagonal line length, which could be used as estimator/other-name for its characterizing adjectives 'deep', 'cool' and 'slow' with which it is traditionally associated. 
Table 1: Consolidated (average \pm std) RQA measures of PPa series for healthy individual, across eight days period in prominent humor interval.

\begin{tabular}{|c|c|c|c|c|c|c|c|c|}
\hline \multirow{3}{*}{ Interval } & RR & DET & L_mean & L_max & $\begin{array}{c}\text { ENTR } \\
\text { (diagonal) }\end{array}$ & LAM & TT & Vmax \\
\cline { 2 - 9 } & $(\%)$ & $(\%)$ & (beats) & (beats) & - & $(\%)$ & (beats) & (beats) \\
\hline \multirow{2}{*}{$\mathrm{Kp}$} & 18 & 98 & 16.06 & 142.75 & 3.18 & 85 & 7.69 & 49.13 \\
& \pm 12 & \pm 2 & \pm 9.05 & \pm 82.04 & \pm 0.54 & \pm 14 & \pm 4.33 & \pm 23.39 \\
\hline \multirow{2}{*}{$\mathrm{Pt}$} & 3 & 79 & 4.96 & 22.50 & 1.76 & 35 & 3.37 & 13.25 \\
\hline $\mathrm{Vt}$ & \pm 4 & \pm 33 & \pm 3.32 & \pm 20.83 & \pm 1.00 & \pm 34 & \pm 1.28 & \pm 16.46 \\
\hline
\end{tabular}

Table 2: Consolidated (average \pm std) RQA measures of PPa series, in Kp interval, for diseased individual - Herpes Zoster infection condition, over four days period.

\begin{tabular}{|c|c|c|c|c|c|c|c|c|}
\hline \multirow{3}{*}{ Interval } & RR & DET & L_mean & L_max & ENTR (diagonal) & LAM & TT & Vmax \\
\cline { 2 - 9 } & $(\%)$ & $(\%)$ & (beats) & (beats) & - & $(\%)$ & (beats) & (beats) \\
\hline \multirow{3}{*}{$\mathrm{Pt}$} & 5 & 89 & 6.10 & 26.56 & 1.86 & 35 & 3.03 & 12.11 \\
& \pm 6 & \pm 14 & \pm 4.26 & \pm 27.76 & \pm 1.03 & \pm 38 & \pm 1.24 & \pm 14.74 \\
\hline
\end{tabular}

Table 3: Pearson's correlation between average values of RQA measures for healthy individual in respective humor intervals (Table 1) with those for group of individuals (13) in Pt interval period (Table 2).

\begin{tabular}{|l|c|c|c|}
\hline $\begin{array}{l}\text { Table 1, Marker RQA } \\
\text { measures of PPa in } \\
\text { respective humor interval. }\end{array}$ & Kp interval & Pt interval & Vt interval \\
\hline $\begin{array}{l}\text { Table 2, RQA measures } \\
\text { for group of individuals of } \\
\begin{array}{l}\text { PPa in Pt interval with Pt } \\
\text { aggravation. }\end{array}\end{array}$ & 0.657 & 0.998 & 0.949 \\
\hline
\end{tabular}

The qualifiers for describing $\mathrm{Kp}$ humor may also be interpreted to convey high determinism as evident from the table. Although most points for Kp humor contribute to diagonal line structures, DET- $98 \%$, however the relatively higher level of entropy conveys more complexity, indicating higher textural details of the plot structure. Similarly Pt humor qualified as 'hot' 'jerky' and 'high in tension', can be interpreted as having lower level of determinism as indicated by the table. On a relative level, Pt humor has equally low richness and complexity as conveyed by the lower entropy value. Vt humor, described as 'fast', 'cold' and 'low in tension' has identifiably, measure values between $\mathrm{Kp}$ and $\mathrm{Pt}$ humor, although none observably prominent with respect to its characteristic features.

Vertical structures result in RP when the state of the signal in the phase-space trajectory is unchanging or evolving slowly over time [16]. All three humors have reasonably lower trapping time (TT) values compared to the mean diagonal line lengths (Lmean) which probably indicates that the signal states of the three humors are in constant flux.
Among the three humors, Pt humor is not only known to peak at noon, but also prominent on fasting or empty stomach. To physiologically isolate and identify the humor in individuals and compare the results with those above, a group of 13 healthy individuals selected randomly and belonging to age group of 21 to 40 years have been considered. Subsequent to informed consent, fasting pulse was obtained at Pt humor peak time (P_pulse) - $1.00 \mathrm{pm}$, ensuring at-least $4 \mathrm{hrs}$. fasting. For healthy individuals this reasonably ensures the dominance of Pt humor allowing for cross-verification of results with those obtained for a single healthy individual. Table 2 lists the consolidated results (average \pm std) of RQA measures for, $\mathrm{PPa}$, of P_pulse for the 13 individuals. On comparing the RQA measures for 13 individuals with those of single healthy individual, it is realised that the average measures for the two lie within a reasonable range, and correlate by a factor of 0.998 .

Table 3 above indicates the strong positive correlation (3rd col.) between the averaged RQA measures of Pt humor for healthy individual with those for group of individual (0.998). The 2nd col. of table depicts milder correlation of average RQA measures for healthy individual in $\mathrm{Kp}$ interval with 
those for group of individual in Pt interval period (0.657). However the larger correlation values in last col. need further study in light of observing pulse condition with Vt humor isolated and aggravated physiologically. Moreover the larger value in $4^{\text {th }}$ col $(0.949)$ could also be interpreted as an indicator of similar characterizing features displayed by $\mathrm{Vt}$ and Pt humor, traditionally described as 'random in rate' \& 'fast' and 'hot' \& 'jerky' respectively.

\section{CONCLUSIONS}

The primary objective of this study was to explore signal processing measures for identifying the dominant humors $\mathrm{Kp}$, Pt and $\mathrm{Vt}$, in the radial pulse signal, as identified by a TIM physician using sense perception. Radial pulse signal has been sensed using a unique capacitance based sensing mechanism for obtaining signal variations as apparent to the sense perception. RQA measures for pulse signal logged in prominent humor intervals reflect reasonable variations according to the qualitative description for the respective humors as reported in TIM literature. RQA measures of pulse signal for healthy individual, in dominant humor interval period, act as markers. These marker results are observed to correlate with a factor of 0.998 , with those for a group of healthy individuals in dominant Pt humor interval period.

Although RQA measures for Pt humor compare well, measures for $\mathrm{Kp}$ and $\mathrm{Vt}$ humor have not been verified. Present study has been conducted considering a sample group of 13 individuals, which needs to be improved upon for making the results statistically relevant. Similar study which will identify physiological conditions for $\mathrm{Vt}$ and $\mathrm{Kp}$ humor aggravation needs to be undertaken. Unlike the present study which explores pulse amplitude recurrence, use of signal processing tools which analyse the time domain series at native scale need to be explored for obtaining results closer to TIM perspective. Efforts towards rationalization and analytical study of traditional methods will help towards realization of low cost, non-invasive, value-added diagnostic tool for physicians.

\section{ACKNOWLEDGEMENTS}

Authors are highly grateful and thankful to the support and guidance that they have received from Dr. Prasanna Kelkar (MD physician), practicing TIM expert based in Mumbai. Author-1 acknowledges support and encouragement received from management and principal of the parent institute, D. J. Sanghvi COE, VileParle, Mumbai, where most of the experimental work was carried out.

\section{REFERENCES}

[1] Lad, V., 2005. Secrets of the Pulse: The ancient art of Ayurvedic pulse diagnosis, Delhi: Motilal Banarsidass

[2] Amber, R., and Brooke, B., 1993. Pulse Diagnosis Detailed interpretations for Eastern \& Western Holistic Treatments, USA: Aurora Press.

[3] Joshi, R. R., 2005. Diagnostics Using Computational Nadi Patterns. Mathematical and Computer Modelling, 2005;41: 33-47. doi: 10.1016/j.mcm.2004.05.002

[4] Joshi, A., Chandran, S., Jayaraman, V. K., and Kulkarni, B. D., 2007. Arterial Pulse System: Modern Methods For Traditional Indian Medicine. 29th Annu Int Conf of the IEEE EMBS, 2007:608-611.

[5] Thakker, B., Vyas, A. L., and Tripathi, D. M. 2010. Radial pulse analysis at deep pressure in abnormal health conditions. 3rd Int Conf on BMEI 2010, 2010;3:10071010. doi : 10.1109/BMEI.2010.5639735
[6] Sareen, M., Abhinav, Prakash, P., and Anand, S., 2008. Wavelet Decomposition and Feature Extraction from Pulse Signals of the Radial Artery. ICACTE '08 2008:551-555. doi : 10.1109/ICACTE.2008.66.

[7] Abhinav, Sareen M, Kumar M, Anand S, Salhan A, and Santhosh Jayashree, 2008. Nadi Yantra: A Robust System Design to Capture the Signals from the Radial Artery for Non-Invasive Diagnosis. 2nd Int Conf on Bioinformatics and Biomedical Engineering 2008, 2008;1387-1390. doi: 10.1109/ICBBE.2008.676

[8] Department of AYUSH 2011, AYUSH in India 2010. New Delhi, INDIA: Planning and Evaluation Cell, Department of AYUSH, 22nd Issue, July 2011. Available from: http://indianmedicine.nic.in/ . [Accessed : Dec. 2013]

[9] National Institute of Ayurveda, Department of AYUSH, Ministry of Health \& FW, Govt. of INDIA 2014, Ayurveda Concepts [Online] Department of AYUSH, Govt. of INDIA. Available from: http://www.nia.nic.in/ [Accessed : 3rd March 2014]

[10] Joshi, P., and Daruwala, R.. 2013. Investigations into TIM perspective of Radial Pulse Analysis. IEEE Pointof-Care Healthcare Technologies (PHT), 2013; 80-84. doi:10.1109/PHT.2013.6461289

[11] Joshi, P., Kelkar, P., and Daruwala, R., 2013. Radial pulse analysis for circadian cycle evaluation. IEEETENCON 2013- Region 10 Conference, 2013;1-4 doi:10.1109/TENCON. 2013.6719034

[12] Joshi P, and Daruwala R. 2013. Objective evaluation of radial pulse signal, India Conference (INDICON), 2013 Annual IEEE, 2013;1-4. doi:10.1109/INDCON.2013.6726081

[13] Dahanukar, S. and Thatte., U., 2005. Ayurveda Unravelled New Delhi, India : National Book Trust.

[14] Guyton, A. C., and Hall, J. E., 2006. Textbook of Medical Physiology, 11th Ed., Elsevier Saunders.

[15] Costa, M., Goldberger, A. L. and Peng, C. K., 2005. Multiscale Entropy Analysis of Biological Signals. Physical Review 71, $021906 . \quad$ doi: 10.1103/PhysRevE.71.021906.

[16] Marwan, N., Romano, M. C., Thiel, M., Kurths, J., 2006. Recurrence plots for the analysis of complex systems. Physics Reports 438, 237 - 329 , doi : 10.1016/j.physrep.2006.11.001

[17] Webber, C. L., Zbilut, J. P. Jr., 2013. Recurrence Quantification Analysis of Nonlinear Dynamical Systems. In Riley, M., A., \& Van Orden, G., C.,(Eds.), Tutorials in contemporary nonlinear methods for the behavioral sciences (p. 26-94). Retrieved February 25, 2013,

from http://www.nsf.gov/sbe/bcs/pac/nmbs/nmbs.jsp.

[18] Marwan, N., 2011. Cross Recurrence Plot Toolbox. Version 5.16 (R28.12) 18-May-2011. Copyright (c) 2008-2010. Norbert Marwan, Potsdam Institute for Climate Impact Research, Germany. Available from : http://www.pik-potsdam.de , [Accessed: Jan 2013].

[19] Task Force of the ESC and NASPE 1996, Heart rate variability: Standards of measurement, physiological interpretation and clinical use. Circulation, 93:10431065.

[20] Schinkel, S., Dimigen, O., and Marwan, N., 2008. Selection of Recurrence Threshold for Signal Detection. Eur. Phys. J. Special Topics 164, 45-53, doi: 10.1140/epjst/e2008-00833-5. 